\title{
A Comparative Study of Binarization Techniques for Enhancement of Degraded Documents
}

\author{
Chinni Bhargavi S \\ Student \\ Department of Information Science \\ PESIT \\ Bangalore-5600, INDIA
}

\author{
B Priyanka \\ Student \\ Department of Information Science \\ PESIT \\ Bangalore-5600, INDIA
}

\author{
Mamatha $\mathrm{H} \mathrm{R}, \mathrm{PhD}$ \\ Professor \\ Department of Information Science \\ PESIT \\ Bangalore-5600, INDIA
}

\begin{abstract}
Documents are archived and preserved in large quantities worldwide. Electronic scanning is a common approach in handling such materials to facilitate public access to them. But the resulting images are often difficult to read, also have low contrast, and are corrupted by different artifacts. Enhancement of image is normally based on minor deterioration in modern documents to improve optical character recognition. It often ignores cases, such as those typical of historical and other highly degraded documents. Separating background and foreground can make an image readable. The initial approach is using local threshold where an average threshold value is used for sub images. The next approach is using Global thresholding where only single threshold value is used for an entire image. The third approach is combining both the algorithms Local and Global which is Hybrid Binarization where global thresholding is applied and Local thresholding is applied to parts where thresholding is to be done. The last approach being Iterative Global Thresholding which calculates the threshold iteratively and performs thresholding. Filtering is done to remove noises using methods like Smoothing and Sharpening and Peak to signal noise ratio is found before and after to check the degree of enhancement.
\end{abstract}

\section{General Terms}

Image processing, Binarization, Grey Scale conversion, Filtering.

\section{Keywords}

Hybrid binarization, Global thresholding ,local thresholding, Iterative Global Thresholding, Peak to Signal Noise ratio.

\section{INTRODUCTION}

Degraded documents are preserved in large quantities for their extensive usage in these days. Electronic scanning is a common approach in handling such materials to facilitate public access to them. But the resulting images are often difficult to read, have low contrast, and are corrupted by various artefacts. For example, the original document may be faded, washed out, damaged, crumpled, or otherwise difficult to discern. Moreover, it may contain mixed handwriting in one or many languages, typed or printed text, or even tables, pictures, or diagrams. Machine-printed text may have been produced using different technologies with different quality. Accordingly, comprehensibility and readability need to be increased.

A well known technology like the Optical character Recognition (OCR) which enables you to convert different types of documents, such as scanned paper documents, PDF files or images captured by a digital camera into editable and searchable data does not hold good for the documents that are not enhanced. Hence, it is necessary to do suitable enhancement for the degraded image.

Image enhancement is basically improving the interpretability or perception of information in images for human viewers and providing 'better' input for other automated image processing techniques. The main objective of image enhancement is to modify attributes of an image to make it more suitable for a given task and a observer. In this process, one or more attributes of the image are changed. The selection of attributes and the way they are modified are specific to a given task. The enhancement system in this project produces a document image that can be viewed in different ways using different methods for 4 different processes. A common method converting the given image to a greyscale image is done and is taken as an input for the further step. Next step being Binarization/Hybrid Binarization for the separation of pixel values into two collections, white as background and black as foreground. In document which have same contrast delivery of background and foreground, global method has found to be best technique. In documents which are degraded, where extensive background noise or difference exists i.e. there exists many pixels that cannot be easily categorized as foreground or background. In these cases, local method has significant over available techniques. Sometimes both global and local thresholding (Hybrid Binarization) for better results. These Binarization process are carried out using two threshold methods which are Otsu and IGT(Iterative Global thresholding) comparing with different types of threshold like Threshold Binary, Threshold Binary Inverted ,Truncate ,Threshold to zero ,Threshold to zero inverted. A PSNR (peak to signal noise ratio) is calculated after this process to find out the noise present in the image comparing with the original image after which filters are added to remove the noise. Image de-noising is an important image processing task, both as a process, and as a component in other processes. The general process of filtering and applying masks is consists of moving the filter mask from point to point in an image. These are applied on image for different purposes. The most common two uses are as following: 1. Filters are used for noise reduction and Blurring 2. They are also used or edge detection and sharpness. PSNR is found after these filters are added before and after the filtering methods and compared for different document images for different purposes. Below shows the different techniques for binarization

\section{LITERATURE REVIEW}

A specialized processing in order to remove background noise and become more legible is proposed in [1] by Ergina Kavallieratos and Efstathios Stamatatos that using a hybrid binarization approach for improving the quality of old documents using a combination of global and local thresholding. First, a iterative global thresholding technique specifically designed for old document images is applied to the entire image, iteratively with respective steps. Then, the image areas that still contain background noise are detected and the same technique is re-applied to each area separately. Thereby 
achieving better adaptability of the algorithm in cases where various kinds of noise coexist in different areas of the same image and avoiding the computational and time cost of applying a local thresholding in the entire image. An efficient binarization technique proposed by Jagroop Kaur et.al.,.in [2] is the image binarization is the process of separation of pixel values into two collections, white as background and black as foreground. Thresholding is a well-known technique for binarization of document images. Its further divide into the global and local thresholding technique. In document which has same contrast delivery of background and foreground, global method of thresholding has found to be best technique. In degraded documents, where extensive background noise or difference exists i.e. there exists many pixels that cannot be easily categorized as foreground or background. In these cases, local thresholding has significant over available techniques.

An efficient filtering technique is presented [3] proposed by Abdenour Sehad et.al.,. Here the dominant slant angle of the document image script is computed by using the Fourier transform. Then, this angle is used within a weighted sum of angles in a Gabor filter bank in order to capture more efficiently the document image foreground text.

\section{BINARIZATION TECHNIQUES}

Image binarization is a fundamental research theme in image processing and an important method in image recognition. It is not very easy to select the corresponding threshold for each image of different types. The simplest way is to choose a suitable threshold value, and classify the pixels with values above this threshold as white, and other pixels as black. The problem then is to select the correct threshold. In many cases, finding one threshold compatible to the entire image is not very easy, and in many cases even impossible. Types of thresholding done to binarize an image is discussed below.

\section{OTSU'S METHOD (THRESHOLD):}

Otsu's thresholding method involves iterating through all the possible threshold values and calculating a measure of spread for the pixel levels each side of the threshold, i.e. the pixels can either fall in background or in foreground. The aim is to find the threshold value where the sum of foreground and background spreads is at its minimum. In Otsu's method an exhaustively search for the threshold that minimizes the intra-class variance (the variance within the class), as a weighted sum [4] of variances of both the classes:

$$
\sigma_{w}^{2}(t)=\omega_{1}(t) \sigma_{1}^{2}(t)+\omega_{2}(t) \sigma_{2}^{2}(t) \rightarrow
$$

Weights are the probabilities of both separated by a threshold $t$ and are variances

$\mathrm{Eq}(2)$ shows that minimizing the intra-class variance is the same as maximizing inter-class variance:

$$
\sigma_{b}^{2}(t)=\sigma^{2}-\sigma_{w}^{2}(t)=\omega_{1}(t) \omega_{2}(t)\left[\mu_{1}(t)-\mu_{2}(t)\right]^{2} \rightarrow \text { Eq (2) }
$$

expressed in class means $\mu_{i}$ and probabilities $\omega_{i}$.

The class probability $\omega_{1}(t)$ is calculated from the histogram as $t$

$$
\begin{aligned}
& \omega_{1}(t)=\Sigma_{0}^{t} p(i) \longrightarrow \mathrm{Eq}(3) \\
& \text { while the class mean } \mu_{1}(t) \text { is shown }
\end{aligned}
$$

$$
\mu_{1}(t)=\left[\Sigma_{0}^{t} p(i) x(i)\right] / \omega_{1} \longrightarrow \mathrm{Eq}(4)
$$

\section{LOCAL THRESHOLDING TECHNIQUE}

An approach to handling situations in which single value thresholding will not work is to divide an image into sub images and threshold these individually. Since the threshold for every pixel depends on its location within an image this technique is said to be local. A Grayscale image is taken as an input and local thresholding

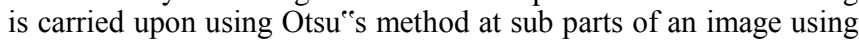
Threshold Binary for better results. The method of evaluation and region sampled vary between applications. Local thresholding which is at a pixel level (in comparison with neighboring pixels) can yield highly superior results compared to global thresholding, specially for images with varying levels of regional contrast differences.

Since the threshold for every pixel depends on its location within an image this technique is said to local. The image below shows an example of working using local thresholding in Fig 1

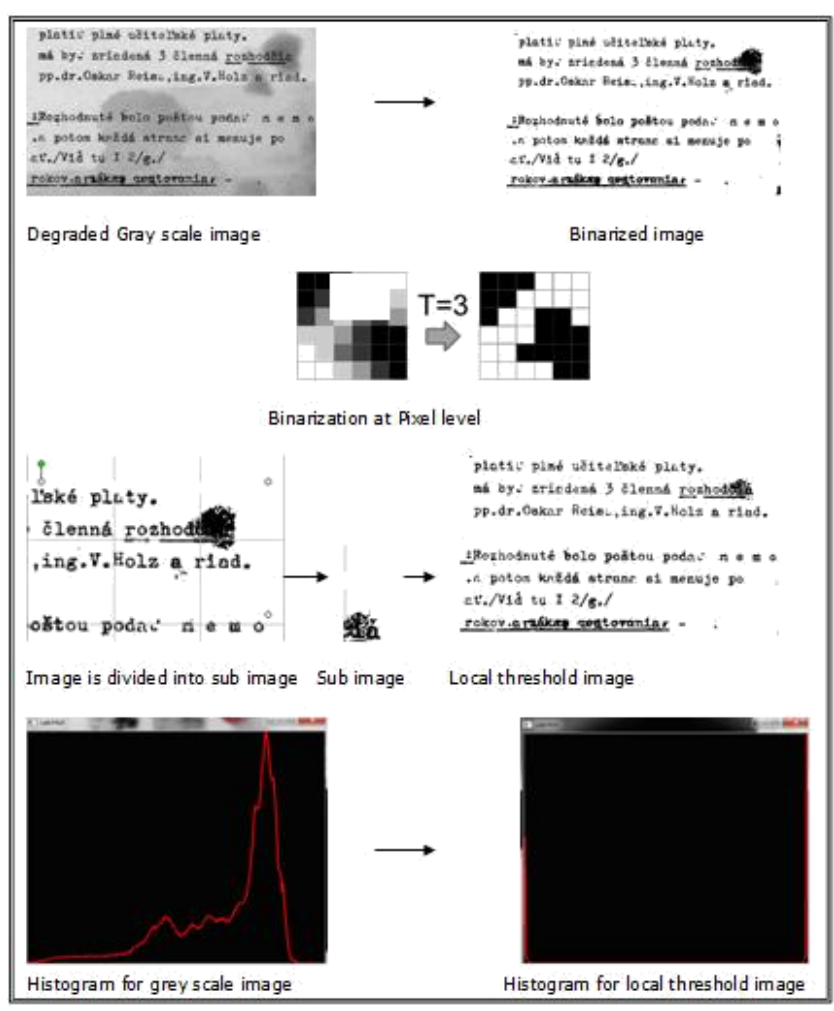

Fig(1) Example for working of Local thresholding

\section{GLOBAL THRESHOLDING TECHIQUE}

Partition the image histogram using a single global threshold .The results of this technique very strongly depends on how well the histogram can be partitioned. Input is taken as Grey scale image and its undergone a Global thresholding technique with Otsu" $s$ method. A clear distinction of the modes in the histogram is witnessed. High-intensity pixels are of interest, where as low intensity are not.Global thresholding example is shown below 


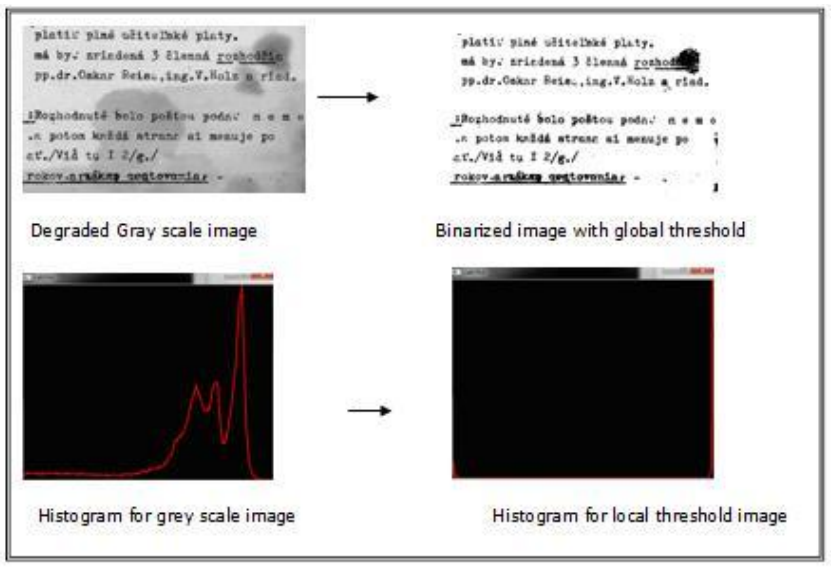

Fig(2) Example of Global thresholding

\section{HYBRID BINARIZATION TECHNIQUE}

Existing binarization techniques focus either on finding an appropriate global threshold or adapting a local threshold for each area in order to remove strains, smear, uneven illumination etc. A hybrid approach is presented that first applies a global thresholding after that, it identifies the image areas that are more likely to still contain noise. Each of these noisy areas is reprocessed separately to achieve better quality of binarization. Firstly, a technique of global thresholding is applied to the whole image. Later, the image area that still contain background noise are detected and the same technique is re-applied to each noisy area separately. The hybrid framework is summarized as:

- Applying Global Thresholding Algorithm,

- Detection of "Noisy" Areas, and

- Applying Local Thresholding Algorithm to the detected areas

By selecting only specific areas of the image for further thresholding, the cost of applying local thresholding is minimized. Again these images are used to further reduce the computational cost. Therefore, with this approach, much better adaptability of the algorithm is achieved in cases where various kinds of noise coexist on the same image, as in local binarization techniques, while keeping the time cost and computational low, like in global ones, since only a limited number of areas (instead of the entire image) need to be processed separately. Example for working of Hybrid Binarization is shown in the below figure

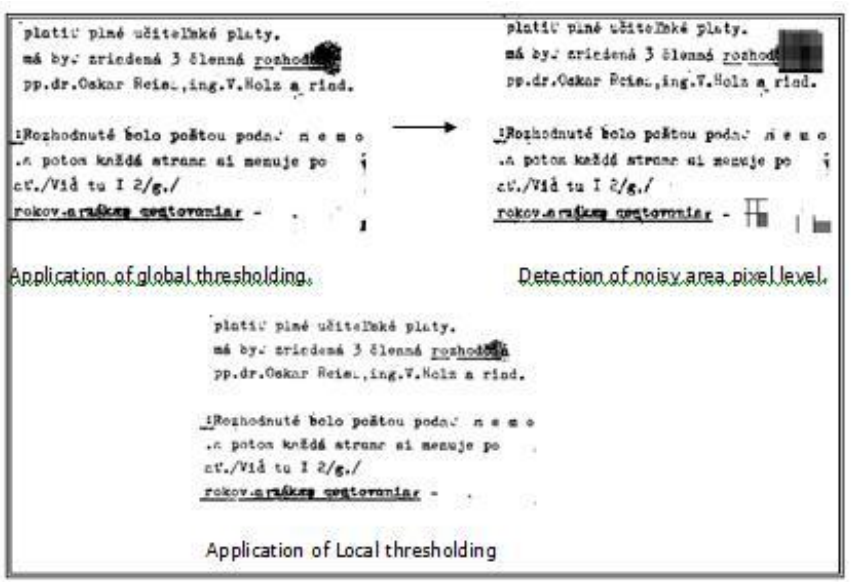

Fig(3) Example for working of Hybrid Binarization

\section{ITERATIVE GLOBAL THRESHOLDING}

The algorithm first applies a global thresholding technique (the IGT), to the document image. The areas that still contain noise are detected and reprocessed separately. In more detail, the proposed algorithm consists of the following steps:

- Applying IGT to the document image.

- Noisy area detection (areas with remaining noise).

- Applying IGT to each detected area separately.

This method is both simple and effective. It selects a global threshold for a document image based on an iterative procedure. In every iteration, the steps performed are:

(i) The average pixel value is calculated, following the below steps,

1. Subtraction of $T i$ from each pixel.

2. The grayscale histogram is stretched so that the remaining pixels to be distributed in all the grey scale tones.

3. Repetition of steps 1-3 till the termination condition is fulfilled

4. Binarization of the final image.

The calculation of the $\mathrm{Ti}$, threshold which is used in i-th repetition, for an $\mathrm{MxN}$ document image, is given by the

$$
T_{i}=\frac{\sum_{x} \sum_{y} I_{i}(x, y)}{M x N} \longrightarrow \mathrm{Eq}(5)
$$

(ii) The average value of pixel is taken and is subtracted from every pixel of the image

$$
\mathrm{I}_{\mathrm{s}}(x, \mathrm{y})=I_{i}(x, y)-T_{i}+1 \longrightarrow \mathrm{Eq}(6)
$$

(iii) Then the histogram is stretched so that the remaining pixels to be distributed in all the grey scale tones.

The iteration is stopped based on the following criterion:

$|T i-T i-1|<0.001$.

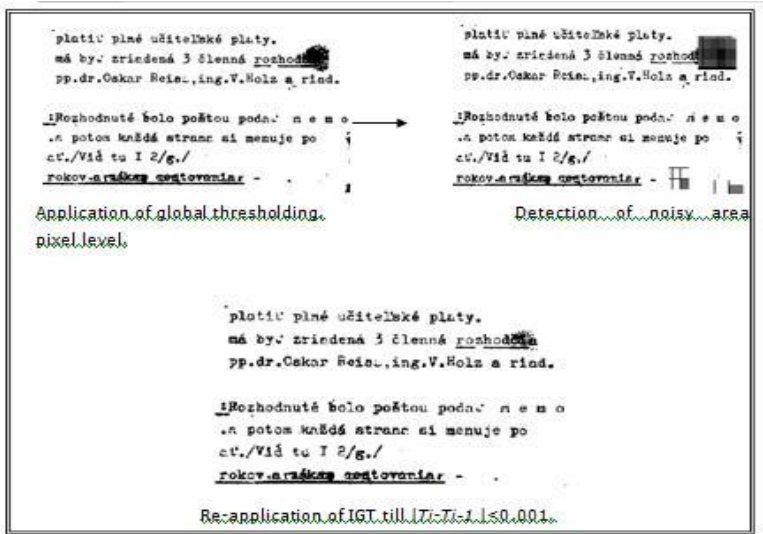

Fig(4) Example for working of IGT 


\section{FILTERING}

Filtering in image processing is a process that cleans up appearances and allows for selective highlighting of specific information. One of the common use of filtering in image processing is to remove blur. Images may be blurred because of moving objects in the frame when the image was taken, file degradation, and other issues. Another use for filtering in image processing is in the handling of images where technicians want to highlight specific objects of interest in the picture. Filtering also have a $2 \mathrm{D}$ filter matrix, and the 2D image. After that, for every pixel of the image, the sum of products is taken. This product gives the result of color value of the current pixel or a neighboring pixel of it, with the value of the filter matrix. The center of the matrix has to be multiplied with the current pixel, other elements of the matrix with corresponding neighbor pixels.

\section{CONCEPT OF MASK}

A mask is a filter. Concept of masking is also known as spatial filtering. Masking is nothing but filtering. This concept just deals with the operation of filtering that is performed directly on the $\mathrm{Eq}(1)$

Filters are for multiple purposes. The most common two uses are as following:

\section{Used for Blurring and noise reduction}

2. Used for edge detection and sharpness

\section{FINDING PSNR}

The term peak signal-to-noise ratio (PSNR) is an expression for the ratio between the maximum possible value (power) of a signal and the power of distorting noise that affects the quality of its representation[5]. Because most of the signals have a very wide dynamic range, (the ratio between smallest and largest possible values of a changeable quantity) the PSNR is usually expressed in terms of the logarithmic decibel scale.

Image enhancement or improving the visual quality of a digital image can be subjective. One person cannot say that one method provides a better quality image. Because of this reason, it is essential to establish quantitative/empirical measures to compare the effects . Using the similiar set of images, various image enhancement algorithms can be compared systematically to identify whether a particular algorithm produces better results.Peak-signal-to-noise ratio is the metric under investigation. If we can show that an algorithm or set of algorithms can enhance a degraded known image to more closely resemble the original, then we can precisely conclude that it is a better algorithm. For the following implementation, let us deal with a standard 2D array of data or matrix. The dimensions of the image matrix which is correct and the dimensions of the degraded image matrix must be identical. The Representation of PSNR is given by

$$
P S N R=20 \log _{10}\left(\frac{M A X_{f}}{\sqrt{M S E}}\right) \longrightarrow \mathrm{Eq}(7)
$$

where the MSE (Mean Squared Error) is given in $\mathrm{Eq}(8)$ :

$$
\begin{gathered}
M S E=\frac{1}{m n} \sum_{0}^{m-1} \sum_{0}^{n-1}\|f(i, j)-g(i, j)\|^{2} \\
\mathbf{f} \text { is the matrix of original image }
\end{gathered} \rightarrow \mathbf{E q ( 8 )}
$$

$\mathbf{g}$ is the matrix of degraded image

$\mathbf{m}$ is the rows of pixel of the images and $i$ is the

index of that row .

$\mathbf{n}$ is the number of columns of pixels of the image and $\mathrm{j}$ is the

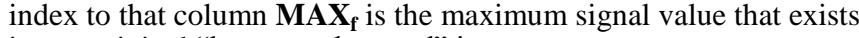
in our original "known to be good" image.

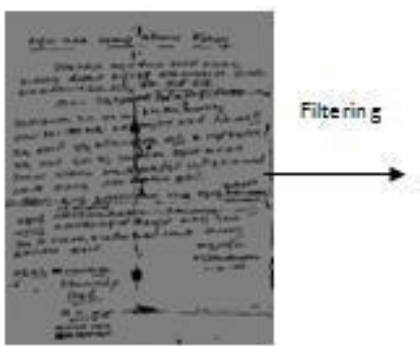

Threshold image

PSNR: 378.107977

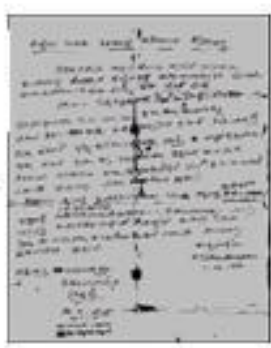

Sharpened image

PSNR: $465.3687 \mathrm{db}$
Fig (6) Working of PSNR

The proposal is that the higher the value of PSNR, the better image of degraded has been reconstructed to match the original image and the better the algorithm. This would occur because we wish to reduce the MSE between images with respect the maximum signal value of the image.

Smoothing, also called blurring, which is frequently used image processing operation. The Gabor filters is one of the advance approaches for image denoising techniques. A low pass filter is used for Smoothing. A low-pass filter is used to smooth an image. This reduces the noise by canceling out rapid variations from pixel . Noise appears at any spots of the image which usually are very different in value from their neighboring pixels. In this filtering techniques for modifying or enhancing an image and emphasize certain features or remove other features. In spatial domain, a 2D Gabor filter is a linear filter whose impulse response is a sinusoidal plane wave modulated by a Gaussian kernel function namely a Gabor wavelet. The Gaussian Function is given as,

$$
g(x)=\frac{1}{\sigma \sqrt{2 \pi}} e^{-\frac{1}{2}\left(\frac{x-\mu}{\sigma}\right)^{2}} \cdot \longrightarrow \mathrm{Eq}(9)
$$

A Gaussian blur effect is typically generated by convolving an image with a kernel of Gaussian values. In practice, its good to use the Gaussian blur"s separable property by dividing the process into two passes. In first pass, a 1D kernel is used to blur the image in only horizontal or vertical direction. In another pass, another onedimensional kernel is used to blur in the rest of the direction. The resulting effect is the similar to convolving a two-dimensional kernel in a one pass, but requires little calculations.

\section{SHARPENING}

Image sharpening refers to any enhancement technique that highlights edges and fine details in an image. In principle, image sharpening consists of adding to the original image a signal that is proportional to a high-pass filtered version of the original image. High-pass filters operate in the same way as low-pass filters: by analyzing the values of each pixel and changing it based on the values of its neighbors. But, its effect is opposite. Instead of an image smoothing, high-pass filters sharpen and bring out minute details.
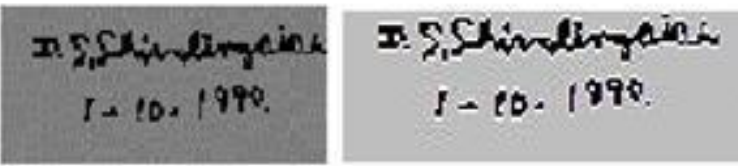
STUDY OF RESULTS OF DIFFERENT METHODS USING

\section{PSNR}

PSNR VALUES FOR LOCAL

THRESHOLDING

\begin{tabular}{|c|c|c|}
\hline \multirow{2}{*}{$\begin{array}{l}\text { SAMPLE } \\
\text { IMAGES }\end{array}$} & \multicolumn{2}{|c|}{$\begin{array}{l}\text { PSNR VALUE IN COMAPRISON } \\
\text { WITH ORIGINAL IMAGE }\end{array}$} \\
\hline & $\begin{array}{l}\text { BEFORE } \\
\text { FILTERING/AFTER } \\
\text { THRESHOLDING }\end{array}$ & $\begin{array}{l}\text { AFTER } \\
\text { FILTERING }\end{array}$ \\
\hline IMAGE 1 & 34.767 & 34.518 \\
\hline IMAGE 2 & 31.058 & 31.012 \\
\hline IMAGE 3 & 36.133 & 35.825 \\
\hline IMAGE 4 & 33.707 & 33.740 \\
\hline IMAGE 5 & 33.457 & 33.459 \\
\hline IMAGE 6 & 33.890 & 33.807 \\
\hline IMAGE 7 & 32.712 & 32.723 \\
\hline IMAGE 8 & 32.069 & 31.996 \\
\hline IMAGE 9 & 36.855 & 36.527 \\
\hline IMAGE 10 & 41.893 & 40.089 \\
\hline
\end{tabular}

The Values shown above in the table represent the PSNR values before and after filtering with an Average value for Local threshold being 170 . The values are same for few samples and change for others depending upon how well the MSE(Mean Squared error ) has reduced. Here we witness three kinds of results

(1) The PSNR slightly decreases implying thresholding gives an optimal result (2) The PSNR increases then the noise has been reduced and image has been enhanced.

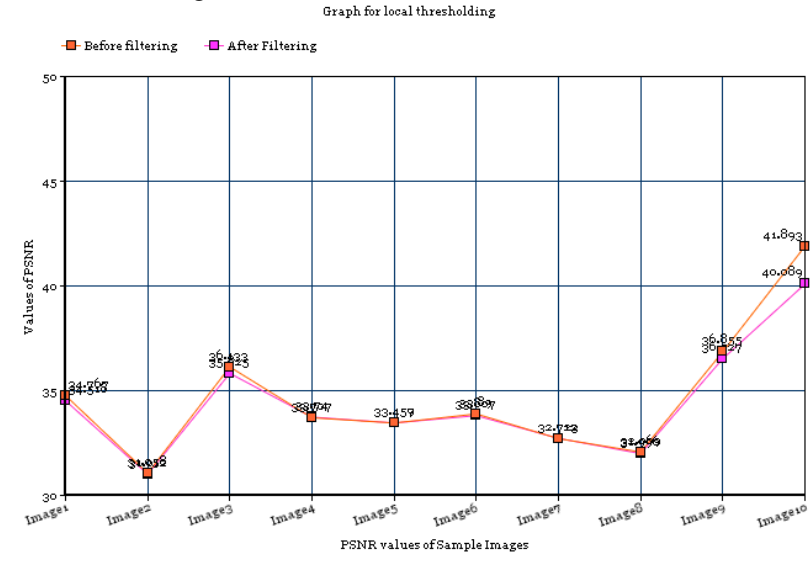

Fig(8) Graph for Global thresholding
The Graph in Fig(8) represents that the values before and after the calculation of PSNR is same, this means optimal result is found at Thresholding stage and no further filtering is required

PSNR VALUES FOR GLOBAL THRESHOLDING

\begin{tabular}{|l|l|l|}
\hline \multirow{2}{*}{ SAMPLE } & \multicolumn{2}{|l|}{$\begin{array}{l}\text { PSNR VALUE IN COMAPRISON WITH } \\
\text { ORIGINAL IMAGE }\end{array}$} \\
\cline { 2 - 3 } IMAGES & $\begin{array}{l}\text { BEFORE } \\
\text { FILTERING/AFTER } \\
\text { THRESHOLDING }\end{array}$ & $\begin{array}{l}\text { AFTER } \\
\text { FILTERING }\end{array}$ \\
\hline IMAGE 1 & 34.299 & 37.838 \\
\hline IMAGE 2 & 38.402 & 40.297 \\
\hline IMAGE 3 & 34.148 & 39.402 \\
\hline IMAGE 4 & 35.086 & 39.323 \\
\hline IMAGE 5 & 31.076 & 32.171 \\
\hline IMAGE 6 & 35.700 & 39.825 \\
\hline IMAGE 7 & 40.367 & 40.367 \\
\hline IMAGE 8 & 40.620 & 40.620 \\
\hline IMAGE 9 & 34.795 & 34.795 \\
\hline IMAGE 10 & 31.895 & 38.642 \\
\hline
\end{tabular}

The Values shown above in the table represent the PSNR values before and

after filtering using Otsu"s method with Threshold Binary for values 0,127.The Values remain same for some samples and change for others depending upon how well the MSE (Mean Squared error) has reduced. Here we witness two kinds of results

(1) If PSNR is same it is considered to have Optimal results with global thresholding (2) If PSNR increases then the noise has been reduced and image has been enhanced.

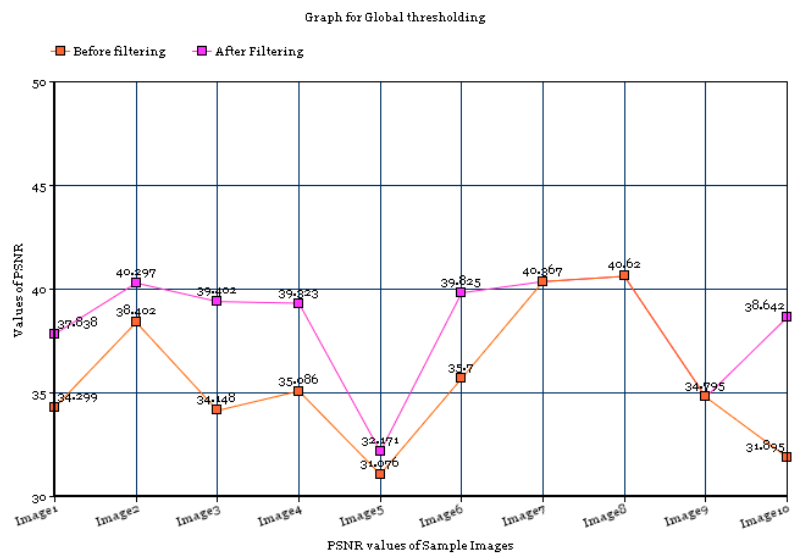

Fig(9) Graph for Global Thrsholding

The Graph in Fig (9)represents the PSNR values before and after filtering using global threshold and the PSNR increases in most cases which implies that filtration will give better results after Binarization. 
PSNR VALUES FOR HYBRID BINARIZATION

\begin{tabular}{|l|l|l|}
\hline \multirow{2}{*}{ SAMPLE } & \multicolumn{2}{|c|}{$\begin{array}{c}\text { PSNR VALUES } \\
\text { IMAGE }\end{array}$} \\
\cline { 2 - 3 } IMAGES & $\begin{array}{l}\text { BEFORE } \\
\text { FILTERING/AFTER } \\
\text { THRESHOLDING }\end{array}$ & $\begin{array}{l}\text { AFTER } \\
\text { FILTERING }\end{array}$ \\
\hline IMAGE 1 & 35.646 & 35.323 \\
\hline IMAGE 2 & 33.684 & 33.520 \\
\hline IMAGE 3 & 36.674 & 35.315 \\
\hline IMAGE 4 & 35.626 & 35.301 \\
\hline IMAGE 5 & 33.840 & 33.616 \\
\hline IMAGE 6 & 35.105 & 34.928 \\
\hline IMAGE 7 & 32.304 & 32.225 \\
\hline IMAGE 8 & 32.069 & 32.001 \\
\hline IMAGE 9 & 36.085 & 36.527 \\
\hline IMAGE 10 & 42.331 & 42.331 \\
\hline
\end{tabular}

The Values shown above in the table represent the PSNR values before and after filtering for Hybrid binarization methods where Local and Global are used with an Average value for Local threshold being 115. The Values remain same for some samples and change for others depending upon how well the MSE(Mean Squared error ) has reduced. Here we witness two kinds of results

(1) The PSNR slightly decreases, it is said to have Optimal results with Local plus Global thresholding and filtering would only hamper the image.

(2) The PSNR increases then the noise has been reduced and image has been enhanced.

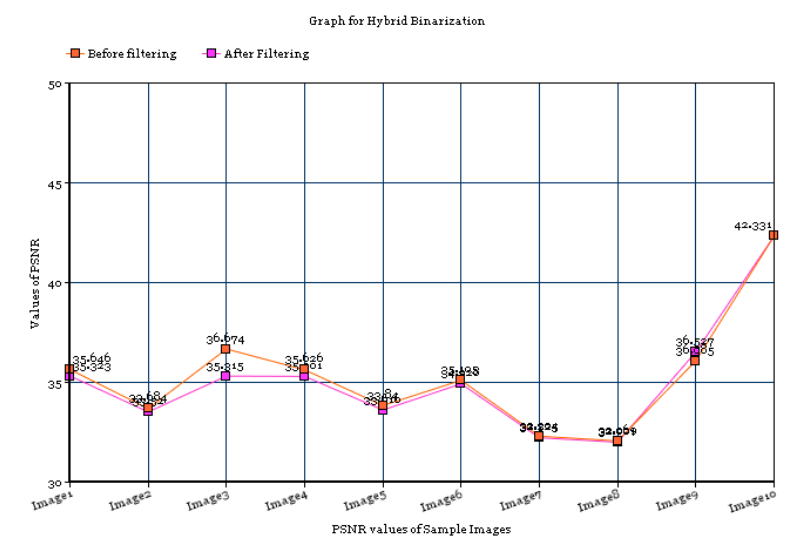

Fig(10)Graph of PSNR values for Hybrid Binarization

The Graph in Fig(10) represents the PSNR values before and after filtering and it implies that local and global thresholding helps in optimum result in enhancement and all the noises are removed in the process itself further filtration gives slight difference in the PSNR values where it increases or decreases.

\section{PSNR RESULTS FOR IGT}

\begin{tabular}{|l|l|l|}
\hline \multirow{2}{*}{ SAMPLE } & \multicolumn{2}{|c|}{$\begin{array}{c}\text { PSNR VALUES } \\
\text { IMAGE }\end{array}$} \\
\cline { 2 - 3 } IMAGES & $\begin{array}{l}\text { BEFORE } \\
\text { FILTERING/AFTER } \\
\text { THRESHOLDING }\end{array}$ & $\begin{array}{l}\text { AFTER } \\
\text { FILTERING }\end{array}$ \\
\hline IMAGE 1 & 36.985 & 36.985 \\
\hline IMAGE 2 & 35.064 & 35.064 \\
\hline IMAGE 3 & 37.402 & 37.402 \\
\hline IMAGE 4 & 37.109 & 37.109 \\
\hline IMAGE 5 & 32.462 & 32.462 \\
\hline IMAGE 6 & 36.090 & 36.090 \\
\hline IMAGE 7 & 33.102 & 33.102 \\
\hline IMAGE 8 & 32.877 & 32.877 \\
\hline IMAGE 9 & 37.730 & 37.730 \\
\hline IMAGE 10 & 42.411 & 42.411 \\
\hline
\end{tabular}

The Values shown above in the table represent the PSNR values before and after filtering for IGT method. The Values remain same for all samples before and after filtering, because results compared with other binarization techniques. The values (a) Increase after filtering indicating that noise has decreased and the image has been enhanced.(b) Values of PSNR remaining same after filtering implies that thresholding technique yields optimal results.(c) Slight decrease in the PSNR values indicate that de-noising technique has not been

satisfactory to that particular image, and only thresholding technique is optimal.

(3) Using IGT which uses de-noising methods within the process itself helps in reducing the cost of applying filters and hence values of PSNR remains same before and after IGT.

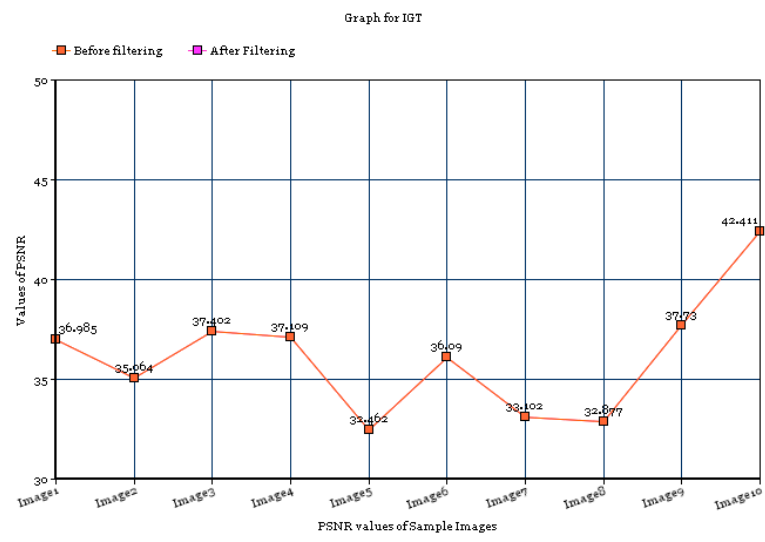

Fig(11) graph for PSNR values for IGT

The Graph in Fig(11) represents PSNR for IGT which implies the value before and after filtering remains the same and no need of filtering since most of the de-noising is done at the binarization stage only. 
The below table shows figures of PSNR values of before and after the filtering techniques for 4 different methods of enhancing a degraded document. The slight variation in values depends on the samples used. The PSNR increases for some images like IMAGE 1,IMAGE 7,IMAGE 10 which shows significant increase in the PSNR values for Global thresholding which implies filtering is necessary . For images like IMAGE 7,IMAGE 5 there is a slight increase in the PSNR for local thresholding and slight decrease in Hybrid Binarization. Therefore it is quite evident that

(1) PSNR values remain same before thresholding for Local, Global and Hybrid Binarization implying that thresholding techniques yield results of same kind but very slight variations.
(2) Applying filters to Local ,Global and Hybrid techniques and finding PSNR after filtering yields different results compared with other binarization techniques. The values (a) Increase after filtering indicating that noise has decreased and the image has been enhanced.(b) Values of PSNRremaining same after filtering implies thatthresholding technique yields optimal results.(c) Slight decrease in the PSNR values indicate that de-noising technique has not been satisfactory to that particular image, and only thresholding technique is optimal.

(3) Using IGT which uses de-noising methods within the process itself helps in reducing the cost of applying filters and hence values of PSNR remains same before and after IGT.

\section{COMPARITIVE STUDY FOR RESULTS OF ALL THE FOUR METHODS}

\begin{tabular}{|c|c|c|c|c|c|c|c|c|}
\hline \multirow[t]{2}{*}{$\begin{array}{l}\text { SAMPLE } \\
\text { IMAGES }\end{array}$} & \multicolumn{2}{|c|}{ LOCAL THRESOLDING } & \multicolumn{2}{|c|}{$\begin{array}{ll}\text { GLOBAL } & \text { THRESOLDING } \\
\text { METHOD } & \end{array}$} & \multicolumn{2}{|c|}{$\begin{array}{l}\text { HYBRID BINARIZATION } \\
\text { METHOD }\end{array}$} & \multicolumn{2}{|c|}{$\begin{array}{l}\text { ITERATIVE GLOBAL } \\
\text { THRESHOLIND }\end{array}$} \\
\hline & $\begin{array}{l}\text { PSNR } \\
\text { VALUES } \\
\text { BEFORE } \\
\text { FILTERING }\end{array}$ & $\begin{array}{l}\text { PSNR } \\
\text { VALUES } \\
\text { AFTER } \\
\text { FILTERING }\end{array}$ & $\begin{array}{l}\text { PSNR } \\
\text { VALUES } \\
\text { BEFORE } \\
\text { FILTERING }\end{array}$ & $\begin{array}{l}\text { PSNR } \\
\text { VALUES } \\
\text { AFTER } \\
\text { FILTERING }\end{array}$ & $\begin{array}{l}\text { PSNR } \\
\text { VALUES } \\
\text { BEFORE } \\
\text { FILTERING }\end{array}$ & $\begin{array}{l}\text { PSNR } \\
\text { VALUES } \\
\text { AFTER } \\
\text { FILTERING }\end{array}$ & $\begin{array}{l}\text { PSNR } \\
\text { VALUES } \\
\text { BEFORE } \\
\text { FILTERIN }\end{array}$ & $\begin{array}{l}\text { PSNR } \\
\text { VALUES } \\
\text { AFTER } \\
\text { FILTERING }\end{array}$ \\
\hline IMAGE 1 & 34.767 & 34.518 & 34.299 & 37.838 & 35.646 & 35.323 & 36.985 & 36.985 \\
\hline IMAGE 2 & 31.058 & 31.012 & 38.402 & 40.297 & 33.684 & 33.520 & 35.064 & 35.064 \\
\hline IMAGE 3 & 36.133 & 35.825 & 34.148 & 39.402 & 36.674 & 35.315 & 37.402 & 37.402 \\
\hline IMAGE 4 & 33.707 & 33.740 & 35.086 & 39.323 & 35.626 & 35.301 & 37.109 & 37.109 \\
\hline IMAGE 5 & 33.457 & 33.459 & 31.076 & 32.171 & 33.840 & 33.616 & 32.462 & 32.462 \\
\hline IMAGE 6 & 33.890 & 33.807 & 35.700 & 39.825 & 35.105 & 34.928 & 36.090 & 36.090 \\
\hline IMAGE 7 & 32.712 & 32.723 & 40.367 & 40.367 & 32.304 & 32.225 & 33.102 & 33.102 \\
\hline IMAGE 8 & 32.069 & 31.996 & 40.620 & 40.620 & 32.069 & 32.001 & 32.877 & 32.877 \\
\hline IMAGE 9 & 36.855 & 36.527 & 34.795 & 34.795 & 36.085 & 36.527 & 37.730 & 37.730 \\
\hline IMAGE 10 & 41.893 & 40.089 & 31.895 & 38.642 & 42.331 & 42.331 & 42.411 & 42.411 \\
\hline
\end{tabular}

\section{CONCLUSION}

In this project we have presented some methods suitable and effective for enhancement of degraded documents by applying effective techniques like Local thresholding, Global thresholding, Hybrid Binarization, Iterative Global Thresholding which shows satisfactory results for Binarization after which remaining noise has been removed by applying filters to the image using methods like Smoothing and Sharpening. A metric to measure the degree of enhancement is found by Peak to Signal Noise Ratio before and after filtering and the comparative results is found to show the degree of enhancement which remains slightly same before and after for various images, and above methods prove optimal for thresholding as a final method and in some cases noise is reduced only after filtering.

\section{FURTHER ENHANCMENTS}

Binarization techniques has been presented that uses local binarization, global thresholding ,combination of the advantages of global and local binarization and IGT and finding the PSNR by which the degree of enhancement can be assessed .As future work, in order to enhance and find the degree of enhancement the following points will be considered.

Applying other thresholds like Niblack ,Mean ,Median ,Sauvola etc for thresholding.

Applying IGT to Hybrid Binarization.

Applying different filters in both spatial and frequency domain to de-noise and compare the PSNR ratio.

Finding white and Black pixel of a binarized image to check the degree of binarization. 


\section{REFERENCES}

[1] Ergina Kavallieratou and Efstathios Stamatatos, "Improving the Quality of Degraded Document Images", DIAL" 06 , August 2006

[2] Jagroop Kaur, Dr.Rajiv Mahajan," A Review of Degraded Document Image Binarization Techniques" International Journal of Advanced Research in Computer and Communication Engineering, Vol. 3, Issue 5, May 2014

[3] Abdenour Sehad, Youcef Chibani and Mohamed Cheriet " Gabor Filters for Degraded Document Image Binarization" 14th International Conference on Frontiers in Handwriting Recognition,2014
[4] Himanshu Makkar, Aditya Pundir "Image Analysis Using Improved Otsu,,s Thresholding Method" International Journal on Recent and Innovation Trends in Computing and Communication ,2014, $2122-2126$

[5] Pooja Kaushik and Yuvraj Sharma "Comparison Of Different Image Enhancement Techniques Based Upon Psnr \& Mse" International Journal of Applied Engineering Research, ISSN 0973-4562 Vol.7 No.11 (2012).

[6] Vavilis Sokratis, Ergina Kavallieratou, Roberto Paredes,and Kostas Sotiropoulos "A Hybrid Binarization Technique for Document Images" Learning Structure and Schemas from Documents, SCI 375,165-179,2011. 